\title{
Validity and reliability of Persian version of Listening Styles Profile-Revised (LSP- R) in Iranian students
}

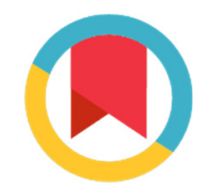

\author{
Zahra Fatehi ${ }^{1}$, Hamid Reza Baradaran ${ }^{2 *}$, Mohamad Asadpour ${ }^{3}$, Mohsen Rezaeian $^{4}$
}

\begin{abstract}
Background: Individuals' listening styles differs based on their characters, professions and situations. This study aimed to assess the validity and reliability of Listening Styles Profile- Revised (LSP- R) in Iranian students.

Methods: After translating into Persian, LSP-R was employed in a sample of 240 medical and nursing Persian speaking students in Iran. Statistical analysis was performed to test the reliability and validity of the LSP-R.

Results: The study revealed high internal consistency and good test-retest reliability for the Persian version of the questionnaire. The Cronbach's alpha coefficient was 0.72 and intra-class correlation coefficient 0.87 . The means for the content validity index and the content validity ratio (CVR) were 0.90 and 0.83 , respectively. Exploratory factor analysis (EFA) yielded a four-factor solution accounted for $60.8 \%$ of the observed variance. Majority of medical students $(73 \%)$ as well as majority of nursing students $(70 \%)$ stated that their listening styles were task-oriented.
\end{abstract}

Conclusion: In general, the study finding suggests that the Persian version of LSP-R is a valid and reliable instrument for assessing listening styles profile in the studied sample.

Keywords: Validity, Reliability, Listening Style Profile, Persian, Iran

Copyright $\odot$ Iran University of Medical Sciences

Cite this article as: Fatehi Z, Baradaran HR, Asadpour M, Rezaeian M. Validity and reliability of Persian version of Listening Styles ProfileRevised (LSP- R) in medical sciences students. Med J Islam Repub Iran. 2017(8 Dec);31.73. https://doi.org/10.14196/mjiri.31.73

\section{Introduction}

Listening is a part in process of communication, without which a break would occur in the communication (1). Listening to others affects how information is remembered and used, and the ways we listen to others powerfully affects individual and relational health and well-being, upward organizational mobility, and how we are seen by others (2). Due to long history of examining individuals' listening styles for more than 50 years a variety of listening concepts and scales are now available (3). Although the goal-directed nature of speaking and listening has long been recognized within the communication discipline, the latter has attracted much less research attention (4). Effec-

Corresponding author: Dr Hamid R Baradaran, baradaran.hr@iums.ac.ir

${ }^{1}$ Education Development Office, Medical school. Rafsanjan University of Medical Sciences, Rafsanjan, Iran.

${ }^{2}$ Center for Educational Research in Medical Sciences, Iran University of Medical Sciences, Tehran, Iran.

${ }^{3}$ Department of Health Services and Health Promotion, School of Health, Rafsanjan University of Medical Sciences, Rafsanjan, Iran.

${ }_{4}$ Department of Epidemiology and Biostatistics, School of Medicine, Occupational Environmental Research Center, Rafsanjan University of Medical Sciences, Rafsanjan, Iran. tive communication is very important in human relations (5). For professions working in the medical fields poor communication is an issue (6) for that the patients' satisfaction is tied to their effective listening (7).

The Listening Styles Profile (LSP-16) was first designed and developed by Watson, Barker, and Weaver (1995) in order to measure an individual's primary preference for listening across in communication discipline (3). Then this questionnaire has been revised (LSP-R) by Bodie and colleagues with higher reliability based on four factors. Therefore, this study aimed to translate this questionnaire into Persian and assess its reliability and validity

$\uparrow$ What is "already known” in this topic:

Sufficient evidence support benefit of effective communication on patients' satisfaction and their care. The Listening Styles Profile-Revised (LSP-R) is the most widely used self-report listening instrument in the communication discipline.

\section{$\rightarrow$ What this article adds:}

Persian version of LSP-R is a valid and reliable instrument for assessing listening styles profile of Iranian students. Majority of medical students $(73 \%)$ as well as majority of nursing students $(70 \%)$ stated that their listening styles had been taskoriented. 
in medical sciences students.

\section{Methods}

The LSP-R questionnaire

The original LSP-R questionnaire includes 24 items and consists of four subscales. Each of these subscales contains six items with a seven point Likert response scale ranging from 'Strongly Agree' to 'Strongly Disagree. Primarily, we obtained a permission from the original developers of LSP-R; then put the questionnaire into forward (English to Persian) and backward (Persian to English) procedures. Four independent translators (two ones for translating English to Persian and two other vice versa) helped us with developing provisional Persian questionnaire. Pre-final version was developed using opinions from a panel of experts in various fields including listening and epidemiology. Final version was developed after administering the questionnaire to 20 students and doing some adaptations for cultural and psychological issues.

The study was carried out in Rafsanjan University of Medical Sciences, Iran during April to July 2015. A sample of medical and nursing students was entered into this study.

\section{Statistical analysis}

\section{Reliability}

For internal consistency Cronbach's alpha coefficients equal or greater than 0.70 was considered satisfactory (8). Test-retest reliability was used to assess the questionnaire's stability estimating the intra-class correlation coefficient (ICC). ICC values of 0.40 or above were considered. Thirty participants completed the questionnaire twice in two-week intervals.

\section{Validity}

We assessed content, face and construct validity of the Persian version of the LSP-R questionnaire using qualitative and qualitative methods.

Content validity: Five experts including one linguists two medical educationists and two health professionals checked the questionnaire for possible syntactic errors, and proper allocation of items. As quantitative measures, content validity index (CVI) and content validity ratio (CVR) were then checked (9). CVI was calculated using a Likert-type ordinal scale (from $1=$ very relevant to $4=$ not relevant). Using experts' opinions CVI was calculated as the proportion of items that received a rating of 3 or 4 (9). For calculating CVR each item was rated as essential, useful but not essential, or not essential (10). After performing validity tests, four items excluded from original version.

Face validity: Initially 10 students were asked to state if they feel any kind of ambiguity in responding the questionnaire. In case they had any difficulty, they were noted to refine the questionnaire. In the quantitative phase, the impact score (frequency $\times$ importance) was measured using the opinions of the recruited students. Impact score equal or greater than 1.5 was considered appropriate (11).

Construct validity: This was checked using expletory factor analysis (EFA) to determine the underlying con-

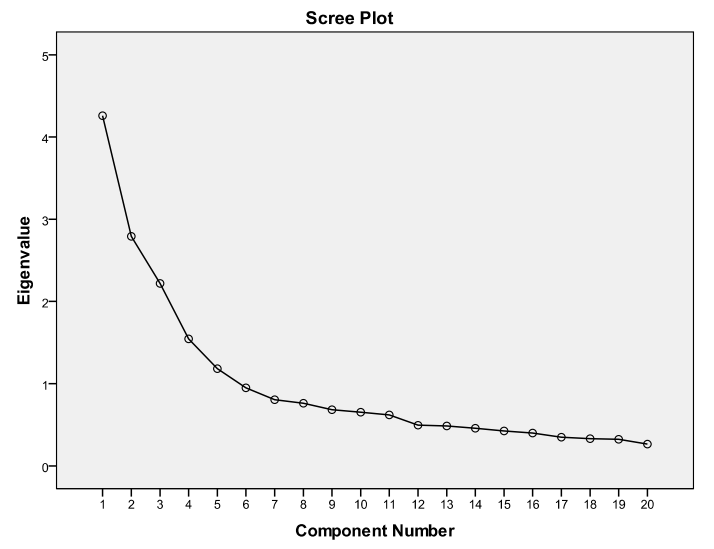

Fig. 1. Scree plot

structs of the questionnaire.

\section{Ethics}

The study protocol was approved by Ethics Committee of Iran and Rafsanjan Universities of Medical Sciences. Written informed consent was obtained from all participants and they were informed about the study objectives and were assured about confidentiality issues.

\section{Results}

The study sample

Two hundred and forty (240) medical and nursing students enrolled in Rafsanjan Medical Sciences University (medicine and nursing schools) randomly selected to participate in this study; $41.2 \%$ of whom were male and $58.8 \%$ female. The mean (SD) age was $21.3 \pm 2.24$ years (Table 1).

\section{Listening Style Profile Revised version}

The descriptive findings for the listening style scores are shown in Table 2. Medical students showed they had a slightly more task-oriented and analytical listening style than nursing; this difference was statistically significant.

\section{Exploratory factor analysis}

Exploratory factor analysis (EFA) was used to measure construct validity and appropriateness of the data for factor analysis was approved $(\mathrm{KMO}$ index $=0.79, \mathrm{p}<0.001)$ (Tables 3-4).

To determine the best structure, the Eigen value greater than one and factor loading equal to or greater than 0.4 were applied (9). The factor analysis described in Table 3 identified the underlying sub concepts that measured the

\begin{tabular}{|c|c|}
\hline Variables & Frequency $(\%)$ \\
\hline \multicolumn{2}{|l|}{ Age (year) } \\
\hline Less than 20 years & $99(41.3 \%)$ \\
\hline $20-25$ years & $132(55.0 \%)$ \\
\hline More than 25 years & $9(3.8 \%)$ \\
\hline \multicolumn{2}{|l|}{ Gender } \\
\hline Female & $141(58.8 \%)$ \\
\hline Male & $99(41.3 \%)$ \\
\hline \multicolumn{2}{|l|}{ Material status } \\
\hline Single & $209(87.1 \%)$ \\
\hline Married & $31(12.9 \%)$ \\
\hline
\end{tabular}




\begin{tabular}{|c|c|c|c|c|}
\hline & $\begin{array}{l}\text { Relational } \\
\text { Mean score (SD) }\end{array}$ & $\begin{array}{l}\text { Analytical } \\
\text { Mean score (SD) }\end{array}$ & $\begin{array}{l}\text { Task-oriented Mean score } \\
\text { (SD) }\end{array}$ & $\begin{array}{l}\text { Critical } \\
\text { Mean score (SD) }\end{array}$ \\
\hline Medical students & $5.3(1.1)$ & $5.5(0.9)$ & $5.6(1.1)$ & $5.1(0.9)$ \\
\hline Nursing students & $5.5(0.5)$ & $5.3(1.0)$ & $5.4(0.8)$ & $5.1(0.9)$ \\
\hline
\end{tabular}

construct of perceived listening effectiveness using five factors. A four-factor solution with 19 items emerged based on Eigen values higher than 1 and loading level of 0.4 or above. The four factors were named according to the underlying construct that related to the items: 1) Relational listening, 2) Analytical listening, 3) Task-oriented listening and 4) Critical listening (Fig. 1).

\section{Reliability}

Test-retest: To test the reliability the internal consistency of the questionnaire was measured using Cronbach's alpha coefficient. To determine the reliability of this scale, 28 students answered the questionnaire items, and after a time interval of 2 weeks, the questionnaire was administered again. The reliability index for the internal consistency using Pearson's moment correlation was 0.718 $(\mathrm{p} \leq 0.001)$.

\section{Internal consistency}

For Internal consistency, Cronbach's alpha coefficient for the whole sample was 0.76 ; for internal factors relational (0.70), factors analytical (0.86), task-oriented (0.70), and factors critical (0.77).

\section{Discussion}

This is the first study that reports on psychometric properties of the Persian version of LSP-R among Iranian medical and nursing students. Since Cronbach's alpha coefficient and intraclass correlation coefficient were acceptable, LSP-R-Persian version is presumed to be valid and reliable to assess listening style. Also, calculated CVI and CVR indicated a reasonable content validity. In the factor analysis five factors with the ability to explain about $60 \%$ of variance of variables. The total variance identified 19 items with acceptable loadings (4\%); therefore the 19-item was considered as the Persian listening style profile in four subscales.

In general, the findings showed promising results and were comparable with most original research findings in English language. Majority of medical students (73\%) and as well as majority of nursing students (70\%) stated that their listening styles were task-oriented, it indicates that these students dislike listening to speakers who take too long to get their point across. This finding is in line with Bodie's report which sated that people who reported high levels of TOL were those who wanted a speaker to remain on task and "get to the point" without wasting time (2).

Table 3. Factor structure of the LSP-R-Persian version using principal component analysis with oblique rotation solution

\begin{tabular}{|c|c|c|c|c|c|}
\hline Questioner & Components & & & & \\
\hline & 1 & 2 & 3 & 4 & 5 \\
\hline Q12 & 0.785 & & & & \\
\hline Q9 & 0.776 & & & & \\
\hline Q11 & 0.772 & & & & \\
\hline Q10 & 0.772 & & & & \\
\hline Q7 & 0.736 & & & & \\
\hline Q8 & 0.728 & & & & \\
\hline Q22 & & 0.802 & & & \\
\hline Q20 & & 0.777 & & & \\
\hline Q19 & & 0.768 & & & \\
\hline Q21 & & 0.740 & & & \\
\hline Q1 & & & 0.826 & & \\
\hline Q2 & & & 0.820 & & \\
\hline Q3 & & & 0.774 & & \\
\hline Q18 & & & & 0.764 & \\
\hline Q16 & & & & 0.753 & \\
\hline Q17 & & & & 0.635 & \\
\hline Q13 & & & & & 0.588 \\
\hline Q15 & & & & & 0.578 \\
\hline Q16 & & & 0.410 & & 0.452 \\
\hline
\end{tabular}

Table 4. Confirmatory factor analysis for modify final questions

\begin{tabular}{|c|c|c|c|c|}
\hline LSP-R subscales & $\begin{array}{l}\text { After Confirmatory factor } \\
\text { analysis }\end{array}$ & $\begin{array}{l}\text { After content validity quantities CVR } \\
(0.51)\end{array}$ & After Face validity & Primary items \\
\hline Relational listening & 4 & 5 & 5 & 6 \\
\hline Analytical listening & 6 & 6 & 6 & 6 \\
\hline Task-oriented listening & 5 & 5 & 5 & 6 \\
\hline Critical listening & 4 & 4 & 4 & 6 \\
\hline
\end{tabular}


In this study, males had a little stronger preference for critical listening style, whereas females had a slightly stronger preference for the Action LS and TOL. However these male-female differences in preferred listening style were not statistically significant. Based on Imhof's idea this gender-difference might be raised due to the wording of the LSP-16's items which prompts males and females to consider different scenarios when responding (12). Therefore, gender differences should be considered while rendering the data obtained from this questionnaire.

Since the samples of this study consisted of medical and nursing students, we urge a caution while generalizing these results to other professionals. Kiewitz et al suggested that the pressures of the workforce may have an impact on people's preferences for listening styles (13). Further studies were suggested to reveal the differences for other health professionals' listening styles.

\section{Limitation}

This study's suffered from some limitations including limited number of samples, and shortage of time for including students from more fields. These limitations hint about generalization of the results.

\section{Conclusion}

In general, the study finding suggests that the Persian LSP-R is a valid and reliable instrument for assessing listening styles profile. Stronger psychometric properties would be reachable in further studies.

\section{Acknowledgments}

None.

Conflict of Interest: None declared.

\section{References}

1. Bodie GD, Crick N. Listening, hearing, sensing: three modes of being and the phenomenology of Charles Sanders Peirce. Commun Theor. 2014;24(2):105-23.

2. Bodie GD, Worthington DL, Gearhart CC. The listening styles profile-revised (LSP-R): A scale revision and evidence for validity. CommunQuart. 2013;61(1):72-90.

3. Bodie GD, Worthington DL. Revisiting the Listening Styles Profile (LSP-16): A confirmatory factor analytic approach to scale validation and reliability estimation. Intl J Listen. 2010;24(2):69-88.

4. Bodie GD. The understudied nature of listening in interpersonal communication: Introduction to a special issue. Intl J Listen. 2011;25(19).

5. Stewart MA. Effective physician-patient communication and health outcomes: a review. Can Med Assoc J. 1995 May 1;152(9):1423-33.

6 Brown T, Boyle M, Williams B, Molloy A, McKenna L, Palermo C, Molloy L. Listening and communication styles of undergraduate occupational therapy students: a cross-sectional study. Br J Occup Ther. 2011, August 2011. 74(Number 8):387-93(7).

7. Arnold CL, Coran JJ. Are you listening healthcare providers? Suggestions for listening skill building education for healthcare providers. Listen Edu. 2011;2(3):5-16.

8. Cronbach L.Coefficient alpha and the internal structure of tests. Psychometrika. 1951, 16: 297-334.

9. Lynn MR: Determination and quantification of content validity. Nurs Res. 1986, 35: 382-385.
10. Lawshe $\mathrm{CH}$. A quantitative approach to content validity. Pers Psychol. 1975, 28: 563-575.

11. Lacasse Y, Godbout C, Series F: Health related quality of life in obstructive sleep apnea. Eur Respir J. 2002, 19: 499-503.

12. Imhof M. Who are we as we listen? Individual listening profiles in varying contexts. Intl J Listen. 2004; 18:36-45.

13. Kiewitz C, Weaver III JB, Brosius H-B, Weimann G. Cultural differences in listening style preferences: $A$ comparison of young adults in Germany, Israel, and the United States. Int J Pub Opin Res. 1997; 9(3):233-247

$4 \quad$ Med J Islam Repub Iran. 2017 (8 Dec); 31:73.
} 